\title{
Object model of technical implementation of calculation of cost of production by method of balance equations
}

\author{
Olga B. Yudakhina, \\ Department of Management \\ National Research Tomsk Polytechnic University \\ Tomsk, Russia \\ liaminor@tpu.ru \\ Timur R. Rakhimov \\ Department of Management \\ National Research Tomsk Polytechnic University \\ Tomsk, Russia \\ rtr@tpu.ru
}

\author{
Yuriy N. Sidorenko \\ Department of Mechanics deformable solids \\ National Research Tomsk State University \\ Tomsk, Russia \\ sid@strelka.ftf2.tsu.ru
}

Veronika V. Zaitseva

Department of Management

National Research Tomsk Polytechnic University

Tomsk, Russia

zaitseva_1205@rambler.ru

\begin{abstract}
One of the most important tasks of management accounting is calculation of cost of production. This paper deals with an embodiment of the balance method calculation of cost based on the object data model. It considers some technical aspects of recording of reference information required for the described object model. A general description of the balance method of calculating of cost is provided. Object model of the balance method is described in detail, including requirements for datasets that serve as input information for balance method and details of the technical implementation of the relevant checks. Examples of program code in Java are provided.
\end{abstract}

Keywords - cost calculation; cost center; in-house services; balance method; object model, object oriented programming, unified modeling language.

\section{INTRODUCTION}

One of the main tasks of managerial accounting is calculation of cost of production. Cost of goods (works, services) of the enterprise is made up of costs associated with the use in process of production of natural resources, raw materials, fuel, energy, fixed assets, labor and other costs of its production and sales expressed in monetary value.

Determination of unit cost of production is one of the basic accounting tasks. Cost of goods is the basis of the solution of a large range of administrative problems. The effectiveness of the organization depends on information about the formation of costs because of the following reasons:

- cost of goods manufactured is the basis for setting the selling price;

- information about cost of goods is used for forecasting and management of production;
- enterprise production costs management is a systematic process of overall production costs formation as well as production costs of individual goods, control over execution of tasks focused on cost reduction and identification of cost reduction potentials. Main elements of cost of goods management system are forecasting and planning, norm setting, accounting and calculation, analysis and control of production costs. They are all interrelated.

One of the most adequate methods of costs calculation, which allows increasing the effectiveness of management decisions, is the method of balancing equations. This method of costs calculation is rarely used by enterprises, due to its complexity of its implementation, but is well described in the works of M.D. Kargapolov [1], Ye.V. Bandakov [2], Ye.S. Zambrzhitskaya [3], N.I. Danilenko [4], A. Apcherch [5].

This paper focuses on development of object model of cost of goods calculation using the method of balancing equations, with emphasis on the procedure of calculation in the context of relevant analytics.

\section{DEFINITIONS}

Prior to description of the model, let us define key notions used in the model.

Branch - a separate structural unit of the enterprise (SSU). If a company has no branches, then branch analytics is absent and, therefore, the model is simplified.

Cost center (CC) - is an object of cost planning and control, which represents technological process, process stage, production unit, group of units which is a process, department or unit (group of units), performing a uniform process or function in the production and sale of goods (works, services). 
Supplier CC - cost center, which produces (performs works, renders services), consumed by other cost centers and sold to third-party contractors.

Consumer CC - cost center that receives and consumes products (works, services), of other enterprise $\mathrm{CC}$. CC can be supplier $\mathrm{CC}$ and consumer $\mathrm{CC}$ at the same time.

Costs - are monetary value (planned or actual) of material, labor, and other resources, consumed in the production in the period under review.

Proper CC costs - costs that can be attributed directly to the $\mathrm{CC}$ at the moment of costs planning or accounting (salaries, materials, etc).

Total CC costs - monetary value (planned or actual) of material, labor and other resources, consumed in the course of economic activity in the period under review, which include proper CC costs, cost of internal resources consumed and the portion of production overhead costs.

Cost item - a group of costs, characterizing processes, necessary for production and sale of good (works, services) and directions of resource spending.

Center of financial responsibility (CFR) - a structural unit of the company that can have a direct impact on the income/expenses of the enterprise in the course of business operations and is responsible for the implementation of the established goals and objectives to it.

Cost element - the primary homogeneous kind of used resources (material, labor, and other), the value of which is transferred to the cost of production, works, or services.

Cost elements are connected with cost items through the aggregation rule, where several elements can be aggregated into a single item. Primary proper costs are planned and accounted for in the context of cost elements.

The model presented in this paper is based on the following assumptions:

- Enterprise organizational structure includes separate structural units (SSU, affiliates), defined in the charter of the organization or selected in the organizational structure;

- Cost centers are objects of planning and accounting for production of goods, works and services.

- Planning and accounting for total CC costs and formation of planned and actual cost of services and types of activities is done in the context of cost elements and cost items.

Planning and accounting of costs is implemented in the context of the following analytics:

- $\mathrm{SSU}$

- CFR (only for primary costs)

- $\mathrm{CC}$

- Cost element (for primary costs) or cost items (for total costs)

\section{- $\quad$ Period}

It is assumed that, aggregation of data is done before calculation of cost of goods. Cost elements are aggregated into cost items, CFR analytics vanishes, which results in formation of aggregate data, which becomes one of the parts of the body of data, used for cost of goods calculation, although it is not absolutely necessary for cost of goods calculation.

To calculate the cost of in-house services in the model information on provision of internal services in the context of a CC is used.

\section{BALANCING METHOD OF COST CALCULATION (BMCC)}

BMCC implies centralized simultaneous calculation of the cost of all services and products of the enterprise, taking into account the mutual provision of services within the enterprise. This method is one of the most accurate methods for calculating the cost of production, though the main difficulty of its introduction is the need to establish a system of algebraic equations which has to be solved. Moreover, For average enterprise (over 500 people) development of thorough cost structure will result in the dimensions of the system of balancing equations of several hundreds and for large enterprises consisting of many SSU, of several thousands. Solving systems of linear algebraic equations (SLAE) of such dimension is only possible with the help of computers and using special computational methods.

Given that the mathematical apparatus of solution of SLAE must be integrated with business content in the context of various analytics, and input data should be read and output data is to be recorded in the database, the implementation of BMCC may seem extremely difficult and, in fact, unrealizable procedure. This paper tries to show that the level of this difficulty is not so great, and if the company has existing planning and accounting systems, the task of implementation $\mathrm{BMCC}$ is quite realizable.

Let us consider in more detail the BMCC model. According BMCC total costs of each CC are defined as the sum of its proper costs and cost of other CCs.

$$
C_{C C i}=P C_{C C i}+\sum_{j} X_{i j}
$$

where, $C_{C C i}$ - total costs of i cost center;

$P C_{C C i}$ - proper (direct) costs of i cost center

$\sum_{j} X_{i j}$ - sum of costs of other cost centers (j) which are applied to cost center i.

Proper costs of $\mathrm{CC}$ for the moment of cost of goods calculation are known. They are costs accumulated at accounts 20, 23, 25, 26, 29 and 44 (in some systems, 30-39 accounts) for the period under review. In case of planning, these are cost planned for specified CC. The difficulty in determining the total cost is to determine the cost of other enterprise CCs, 
formed as a result of in-house services.

In order to determine cost, related to in-house services, it is necessary to define measuring units for each in-house, keep record of services volumes during the accounting period, expressed in physical or synthetic units in the context of Supplier CCs and Consumer CCs. Only in this case volumes of in-house services can be used as a basis for distribution of costs of Supplier CCs.

The volume of production of Suppler $\mathrm{CC}\left(V_{j}\right)$ in accounting period is distributed on Consumer $\mathrm{CCs}\left(V_{i j}\right.$ volume of $\mathrm{j}$ supplier for $\mathrm{i}$-consumer) according to formula 2 :

$$
V_{j}=V_{1}+V_{2}+\ldots=\sum_{i} V_{i j}
$$

Let's define the portion of production volume of $\mathrm{j}$-Supplier $\mathrm{CC}$, that goes to i-Consumer $\mathrm{CC}$ as $K_{j}^{i}$, then the formula will begin to look as follows (3):

$$
K_{j}^{i}=\frac{V_{i j}}{V_{j}}
$$

For the moment of cost calculation (in accounting phase it usually takes place at the closing of the accounting period; in planning phase - after the completion of planning of primary costs and in-house services) this value is already available. Therefore according to $\mathrm{BMCC}$, total costs of i-CC can be determined in the following way (4):

$$
\begin{aligned}
& \mathrm{C}_{C C i}=P C_{C C i}+K_{1}^{i} \cdot C_{C C 1}+K_{2}^{i} \cdot C_{C C 2}+\ldots . K_{N}^{i} \cdot C_{C C N}= \\
& =P C_{C C i}+\sum_{j}^{N} K_{j}^{i} \cdot C_{C C j}
\end{aligned}
$$

It is assumed that in this period the costs are planned / reflected on N CCs. Formally the right side of the equation (4) contains $(\mathrm{N}+1)$ summands. Yet given the fact that in calculating the cost of production Supplier CCs can only be a support, production and service CCs, the number of non-zero terms in the expression (4) is less than $(\mathrm{N}+1)$, and in larger organizations, the structure of which includes a number of SSU, it is substantially less $(\mathrm{N}+1)$.

In the expression (4) at the beginning of the calculation of the cost of production, the known quantities are proper costs and ratios, which represent portions of in-house product (IHP) consumption: $P C_{C C i}$ and $K_{j}^{i}$. If the expression (4) to be written for each of the N CCs, we get a SLAE (5):

$$
\left\{\begin{array}{c}
\mathrm{C}_{\mathrm{CC} 1}=P C_{\mathrm{CC} 1}+K_{1}^{1} \cdot C_{\mathrm{CC} 1}+K_{2}^{1} \cdot C_{\mathrm{CC} 2}+K_{3}^{1} \cdot C_{\mathrm{CC} 3}+\ldots \\
\mathrm{C}_{\mathrm{CC} 2}=P C_{\mathrm{CC} 2}+K_{1}^{2} \cdot C_{\mathrm{CC} 1}+K_{2}^{2} \cdot C_{\mathrm{CC} 2}+K_{3}^{3} \cdot C_{\mathrm{CC} 3}+\ldots \\
\ldots \ldots \\
\mathrm{C}_{\mathrm{CCN}}=P C_{\mathrm{CC} N}+K_{1}^{N} \cdot C_{\mathrm{CC} 1}+K_{2}^{N} \cdot C_{\mathrm{CC} 2}+K_{3}^{N} \cdot C_{\mathrm{CC} 3}+\ldots
\end{array}\right.
$$

The system (5) consists of $\mathrm{N}$ equations, the total costs $\mathrm{C}_{\mathrm{CCi}}$ are unknown, proper costs $P C_{\mathrm{CCi}}-$ are absolute terms, the portions of consumption of IHP $K_{j}^{i}$ - the coefficients of the variables. The solution of system (5) will determine the total cost of each CC, and thus the production cost of each CC:

$$
Z_{C C i}=\frac{C_{C C i}}{V_{C C i}}
$$

where $Z_{C C i}$ - production costs of i-CC.

If the CC directory organized in such a way that one auxiliary or maintenance CC can have more than one type of work, service, or produce more than one type of product, it is necessary to extend the standard BMCC model. To avoid this complication it is advisable to organize $\mathrm{CC}$ directory in such a way where each Supplier CC will have only one type of product or service.

\section{TASKS OF INTRODUCTION OF BMCC}

Therefore the general scheme of BMCC introduction in the enterprise, where there is some form of information system, which allows planning and cost accounting, should look in the following way.

1. Development of reference data, necessary for BMCC implementation, including directory for SSU, CC, cost elements, cost items. Later there will be presented recommendations on the structure of each of the directories, and additional settings to organize correspondences between directories.

2. Determination of the basis for IHP distribution, which is used in BMCC to define coefficients for variables. For that it is necessary to define the list of auxiliary and supporting CCs and measurement units for each of them.

3. On the basis of directories of analytics developed in the above 2 steps, it is necessary to develop functionality for inputs on primary costs and volumes of IHP connected with planned indicators in the context of relevant analytics. Also accounting of costs and volumes of IHP should be organized.

4. Develop functionality for SLAE solution (5).

5. Develop functionality for reporting based on primary and total costs data.

Let us consider in more detail the most important points 


\section{DETERMINATION OF THE BASIS FOR IHP DISTRIBUTION}

Basis of distribution in BMCC is used to determine coefficients for SLAE. Volumes of produced IHP (goods, works, services) are used as the basis of distribution. In the meantime, Supplier CCs of IHP can be auxiliary and supporting and general production CCs, Consumer CCs may by any $\mathrm{CC}$.

Specialists of economic units of the enterprise should define guidelines for measuring volumes of production in physical units for each Supplier CC. In order to record this information in the database it is necessary to develop specific tables, similar to table I.

TABLE I. REFUNITS

\begin{tabular}{l|l|} 
ID, int & Unit, string \\
\hline
\end{tabular}
II.

Then the table of maid data of $\mathrm{CC}$ will transform into table

TABLE II. REFCOSTCENTER

\begin{tabular}{|l|l|l|l|l|}
\hline ID, int & Name, string & ParentID, int & SortOrder, int & UnitID, int \\
\hline
\end{tabular}

Besides reference data, there is transaction data, where every record is specific information referred to a specific moment in time and not to be changed in the future [6]. With regard to $\mathrm{BMCC}$ we define transaction data as planned costs and volumes of IHP, planned for certain SSU, CCs, CFRs, cost elements, cost items in a certain period, as well as accounting data.

For transactional data in the context of IHP it is possible to use the following table structure (tables III, IV):

TABLE III. COSTCENTERSUPPLIER - VOLUMES OF PRODUCTION

\begin{tabular}{|c|c|c|c|c|}
\hline $\begin{array}{c}\text { ID, } \\
\text { int }\end{array}$ & SSP_ID, int & CostCenterID, int & $\begin{array}{c}\text { Period, } \\
\text { Date }\end{array}$ & $\begin{array}{c}\text { Amount, } \\
\text { float }\end{array}$ \\
\hline
\end{tabular}

TABLE IV. COSTCENTERCONSUMER - VOLUMES OF CONSUMPTION

\begin{tabular}{|c|c|c|c|c|c|}
\hline $\begin{array}{c}\text { ID, } \\
\text { int }\end{array}$ & $\begin{array}{c}\text { SSP_ID, } \\
\text { int }\end{array}$ & $\begin{array}{c}\text { CostCenterID, } \\
\text { int }\end{array}$ & $\begin{array}{c}\text { Period, } \\
\text { Date }\end{array}$ & $\begin{array}{c}\text { Amount, } \\
\text { float }\end{array}$ & SupplierID, int \\
\hline
\end{tabular}

Let us point out again that we consider simplified structures, in which details are presented in the form, necessary for description of BMCC. In every specific case, there may be used additional fields and tables or even new table structures, which help implement the described model in more effectively.

\section{STURCTURE OF PRIMARY COSTS OF CCS IN CONNECTION TO BMCC}

General structure of primary costs, used in BMCC was presented in the beginning of this paper. Let us consider it in detail. In general, it is enough for BMCC for every accrual indicator to have the following analytics: period, CC, and SSU in case of complex structure. But management reporting needs are not limited to the calculation of production costs in the context of $\mathrm{CCs}$, so in addition to these analytics for primary costs, it is necessary also to determine such important for management accounting analytics as a cost element and CFR. Then for the planned transaction data table structure can be represented as follows, see Table V.

TABLE V. PlanPRIMARYCOSTS

\begin{tabular}{|c|c|c|c|c|c|c|} 
ID, & $\begin{array}{c}\text { SSP_ID, } \\
\text { int }\end{array}$ & $\begin{array}{c}\text { CostCenterID, } \\
\text { int }\end{array}$ & $\begin{array}{c}\text { CFR_ID, } \\
\text { int }\end{array}$ & $\begin{array}{c}\text { Budget } \\
\text { ElementID, } \\
\text { int }\end{array}$ & $\begin{array}{c}\text { Period, } \\
\text { Date }\end{array}$ & $\begin{array}{c}\text { Sum, } \\
\text { decimal } \\
(16,2)\end{array}$ \\
\hline
\end{tabular}

In the simplest case, we assume that prior to the calculation of the actual cost, the cost structure is already in the form similar to structure of planned costs. Therefore in definition of table with primary costs, we will use "primary costs" name without respect to the type of data (planned/actual).

Since total costs are detailed by cost items, which consist of several cost elements and CFR analytics becomes irrelevant, before calculation of cost it is possible (but not required) to aggregate primary costs in the following structure presented in table VI:

TABLE VI. AGGREGATEDCOSTS

\begin{tabular}{|c|c|c|c|c|c|}
\hline $\begin{array}{c}\text { ID, } \\
\text { int }\end{array}$ & $\begin{array}{c}\text { SSP_ID, } \\
\text { int }\end{array}$ & $\begin{array}{c}\text { CostCenterID, } \\
\text { int }\end{array}$ & $\begin{array}{c}\text { BudgetItemID, } \\
\text { int }\end{array}$ & $\begin{array}{c}\text { Period, } \\
\text { Date }\end{array}$ & $\begin{array}{c}\text { Sum, } \\
\text { decimal } \\
(16,2)\end{array}$ \\
\hline
\end{tabular}

\section{OBJECT MODEL OF SLAE SOLUTION IN THE FRAMEWORK OF $\mathrm{BMCC}$}

There are a number of numerical methods for solving systems of linear equations. They are divided into two main groups - direct and iterative. The direct methods include, for example, the Gauss method, which is a universal method for solving systems of linear algebraic equations.

It is generally accepted that for solving large linear systems with sparse matrices it is more convenient to use iterative methods [7].

For solving SLAE in BMCC it is optimal to use some of the iterative methods, since BMCC matrix is sparce, and the view of recording the system (5) corresponds to the recording of SLAE when converted to the kind used in numerical iterative methods.

Let us consider an iterative method of Gauss-Seidel. For SLAE of the form (7)

$$
\left\{\begin{array}{l}
a_{11} x_{1}+\ldots+a_{1 n} x_{n}=b_{1} \\
a_{n 1} x_{1}+\ldots+a_{n n} x_{n}=b_{n}
\end{array},\right.
$$

Gauss-Seidel method is written as follows (8): 


$$
\left\{\begin{array}{c}
x_{1}^{k+1}=d_{1}+c_{12} \cdot x_{2}^{(k)}+c_{13} \cdot x_{3}^{(k)}+\ldots+c_{1 n} \cdot x_{n}^{(k)} \\
x_{2}^{k+1}=d_{2}+c_{21} \cdot x_{1}^{(k+1)}+c_{23} \cdot x_{3}^{(k)}+\ldots+c_{2 n} \cdot x_{n}^{(k)} \\
\ldots \\
x_{n}^{k+1}=d_{n}+c_{n 1} \cdot x_{1}^{(k+1)}+c_{n 2} \cdot x_{2}^{(k+1)}+\ldots+c_{n(n-1)} \cdot x_{n-1}^{(k+1)}
\end{array}\right.
$$

where $\mathrm{k}$ - number of iteration;

$$
c_{i j}=\left\{\begin{array}{c}
-\frac{a_{i j}}{a_{i i}}, j \neq i \\
0, j=i
\end{array}, d_{i}=\frac{b_{i}}{a_{i i}}, \mathrm{i}=1,2, \ldots . \mathrm{n} .\right.
$$

Comparing recording of SLAE (5) in BMCC with recording (8) of Gauss-Seidel method allows us to conclude that the use of Gauss-Seidel method for solving SLAE (5) requires virtually no additional conversions or conversion factor. Recordings (5) and (8) are structurally identical. Therefore, the Gauss-Seidel method is the most convenient way for solving SLAE (5).

For solving SLAE system (5) by the Gauss-Seidel method, it is possible to use object approach.

One approach allowing effectively to solve issues of modeling of entities and operations on them, is implemented in a modern object-oriented programming (OOP) by means of concepts of classes and objects. We will use further the tools provided by OOP, without paying attention to details of the methodology and principles of the OPP. For code examples in this article uses the Java programming language.
The basic OOP concepts are classes and objects. Class - is an abstract data type. With the help of the class a specific entity can be described (its characteristics and possible actions, links with other classes and classes of objects). After describing the class, you can create an instance of it - the object. Object is a specific representative of its class. [8] Further we consider a model in terms of OOP.

For a visual representation of the classes we use principles of construction of UML-diagrams (Unified Modeling Language), according to which the classes are represented in the form of a rectangle, divided into 3 parts - the upper part presents class name, the middle - list of class attributes (class features and data fields), the bottom part presents a list of class methods (methods here are actions of that class objects "know how" to perform).

All data described above have the same pair of characteristics - SSU, and CC, which are identifying signs of SLAE elements (5) in BMCC. In this case, it makes sense to allocate a separate class that contains the common characteristics of other classes, and to include this class into other classes. Let's call such class CostCenterKey.

In the view of the above, UML-diagram of classes of object model for solving SLAE (5) is shown in the figure 1.

The diagram shows only the basic methods, required for solutions of the system of equations. Let us consider the classes shown in the diagram and the relationship between them.

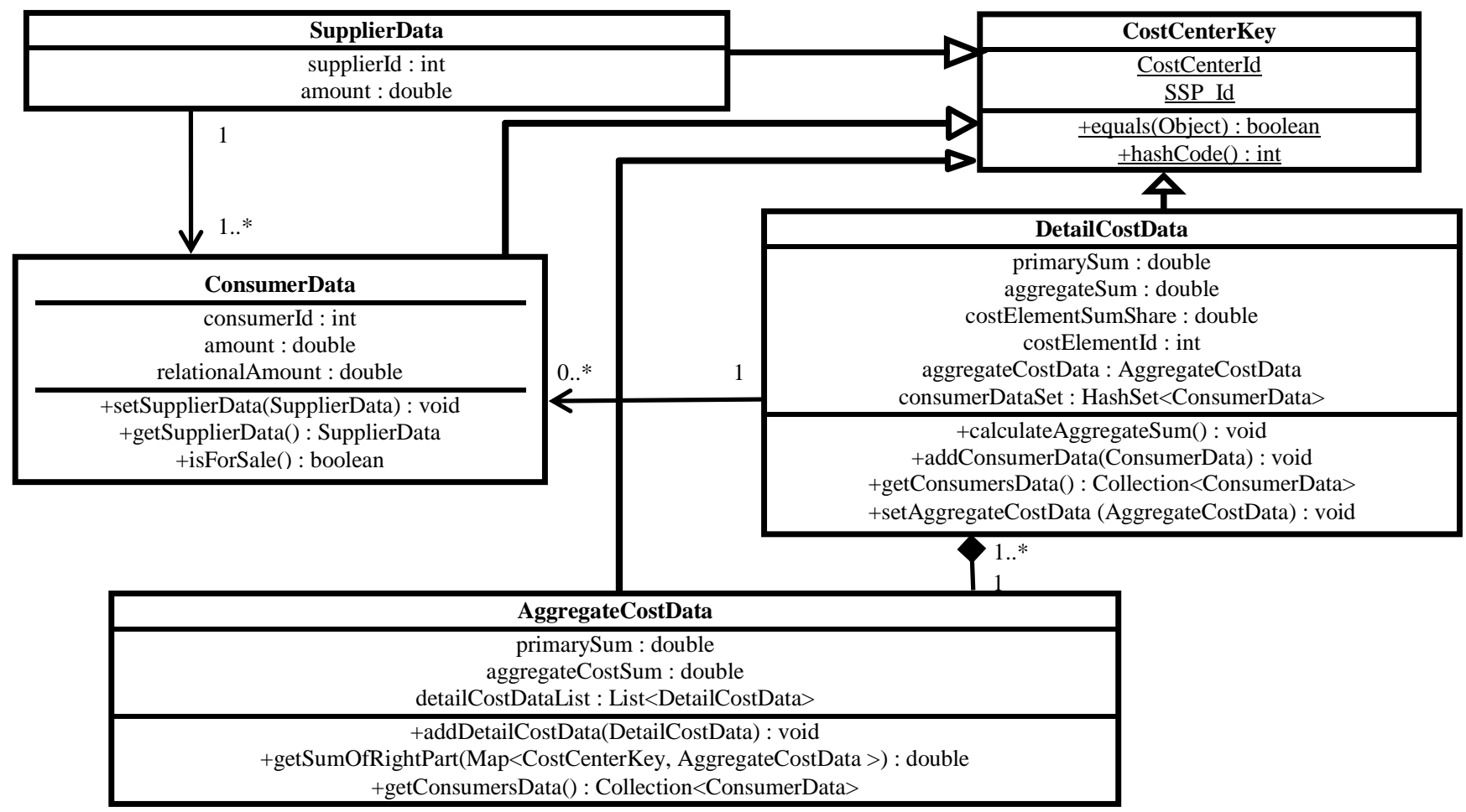

Fig. 1. Object model of technical implementation of calculation of cost of service by methods of balancing equations 
As already mentioned, CostCenterKey class is part of all the other classes. It contains two fields - SSU code and CC code. These codes correspond to the codes of the master data tables in the database - SSP.ID and CostCenter.ID. By these codes at any time the program can obtain the name or other indication of the corresponding attributes. All other classes in the scheme inherit from CostCenterKey class.

In the view of the described UML scheme, the task of calculation of total costs in BMCC may be shown in the following sequence of actions:

1. Calculate from database tables all necessary for BMCC data for the given period. In Java notation internal presentation of calculated data may be shown in the following way:

- from table CostCenterSupplier -> Map<CostCenterKey, SupplierData> suppliersDataMap;

- from table CostCenterConsumer -> Collection<ConsumerData $>$ consumersDataCollection;

- from table AggregatedCosts -> Collection $<$ DetailCostData $>$ costDataCollection.

2. Perform data validation before calculation of costs for fulfillment of conditions, which can not be validated at the stage of user input data. For example, volumes of in-house services in physical units for CC, which doesn't have direct costs are not considered in this paper.

3. Perform aggregation for direct costs. In the database direct costs are stored in SSU-CC-Cost item. When reading this data in the program objects DetailCostData are initialized. In BMCC there are objects with detaisl SSU-CC. Agregation in this case assumes moving from objects DetailCostData to objects AggregateCostData and consists of summing of costs by cost items for a given combination of SSU-CC. In addition, for each object DetailCostData, there is always known the share of its contibution to the sum of costs of a given object AggregateCostData. This is important for further diffirentiation of costs by cost items after calculation of cost of producton.

Map $<$ CostCenterKey, AggregateCostData $>$ aggregateCostDataMap = MatrixesUtil.getAggregateCostDataMap(costDataCollection)

4. Perform calculation of cost of production through BMCC, i.e. solve the system (5) via object model. In Java notation this solution may be written down in the following way:

double difference;

int iterations Num $=0$;

do \{

difference $=0$;

for (AggregateCostData aggrCostData :

aggregateCostDataMap.values()) \{

double sumOfRightPart $=$

aggrCostData.getSumOfRightPart(aggregateCostDataMap); difference $+=$ Math.abs( sumOfRightPart aggrCostData.getAggregatedCommonSum());

aggrCostData.setAggregatedCommonSum(sumOfRightPart); \} iterationsNum++;

\} while (difference > Constants.ACCURACY); /*1.e-10*/

The method getSumOfRightPart of AggregateCostData class me be defined as:

public double getSumOfRightPart (Map<CostCenterKey, AggregateCostData $>$ aggregateCostDataMap)

\{

double sumOfRightPart $=0$;

for (ConsumerData consumerData : getConsumersData()) \{

CostCenterKey id = new CostCenterKey

(consumerData.getSupplierData().getCostCenterId(),

consumerData.getSupplierData().getSSP_Id());

AggregateCostData aggrCostData $=$ aggregateCostDataMap.get(id);

if (aggrCostData != null)

sumOfRightPart+=consumerData.getRelationalAmount ()$*$ fact Data.getAggregatedCommonSum();

\} sumOfRightPart += commonSum; return sumOfRightPart; \}

Presented code fragments almost completely describe the system (5) and provide the implementation of its solution by the Gauss-Seidel method - system (7). In addition, at any time in the code all analytics for each elements of the system are available.

5. Perform differentiation of total costs by cost items.

6. Record results of calculation in relevant table of the database.

\section{RESULTS}

In conclusion the following results of the research may be summarized:

1. Author's object model for calculating the cost of production and services in the enterprises with auxiliary and supporting units is presented.

2. It is rationalized to use in the model the balance method for cost calculating, which currently is one of the most accurate methods of calculation of the cost.

3. It is proposed to use an object-oriented approach to entities modeling, which helps to effectively solve the system of equations used in the balancing method as well as to organize effective storage and processing of business information.

4. A basic structure of the data model is presented. It is focused on the use (as inputs and outputs) of data tables of relational databases, which are the standard for storing business information in today's information systems. 
All of these results have practical significance and contribute to the effective implementation of the model in enterprises by developers who do not have expertise in the field of computational mathematics, with no loss of quality in the implementation of the business requirements of management accounting.

This model has been tested, implemented and successfully operates in the information and control systems in several large and medium-sized enterprises of energy complex of Russia.

The model can be easily transformed for other tasks, which use a system of linear equations with large sparse matrix.

\section{REFERENCES}

[1] M.D. Kargapolov, Balance techniques in economic calculations in the enterprise: Textbook tion allowance; Arkhangelsk, SP D NArFU, 2012,. pp.87.

[2] E.V.Bandakov, "Methods of distribution costs of support units for management purposes: the pros and cons", Problems of Modern Economics: Proceedings of the international. scientific. conf, Chelyabinsk, 2011, pp. 84-87.

[3] E.S. Zambrzhitskaya, A.R Murikova., T.V Kalinin, "Raspredelenie costs of non-productive units matrix method of accounting for the purposes of management accounting as an example of the holding RB-Group", Young scientist, 2012, № 8, pp. 96-100.

[4] N.I. Danilenko, E.S.Zambrzhitskaya, Y.J. Balbardin, "Matrix approach to the allocation of indirect costs and the formation of the full cost of production”, International Accounting, 2015, №16.

[5] A. Upchurch, Management Accounting: Principles and Practice, 2002.

[6] McGraw-Hill, S. P. Parker, Dictionary of Scientific \& Technical Terms, 2003.

[7] V.M. Verzhbitsky, Fundamentals of numerical methods, Moscow: Higher School, 2009, pp. 80-84.

[8] G. Booch, R. A. Maksimchuk, W M. Engle, B. J. Young, J. C.1 Kelly, A. Houston, Object-Oriented Analysis and Design with Applications (UML 2), 2010 .

[9] K.A.Bannova, I.N.Dolgih, A.B.Zhdanova, N.V. Pokrovskaya, "Developing The Competitive Advantage Of Companies And Regions By The Creation Of Consolidated Groups Of Taxpayers", IBIMA, 2015, pp. 834-841. 